\title{
The effect of propensity to trust and perceptions of trustworthiness on trust behaviors in dyads
}

\author{
Gene M. Alarcon ${ }^{1}$ • Joseph B. Lyons ${ }^{1}$ • James C. Christensen ${ }^{1}$ • \\ Samantha L. Klosterman ${ }^{2}$ - Margaret A. Bowers ${ }^{2}$ - Tyler J. Ryan ${ }^{3} \cdot$ Sarah A. Jessup $^{3}$. \\ Kevin T. Wynne ${ }^{4}$
}

Published online: 15 September 2017

(C) W. Montague Cobb-NMA Health Institute (outside the USA) 2017

\begin{abstract}
Research on trust has burgeoned in the last few decades. Despite the growing interest in trust, little is known about trusting behaviors in non-dichotomous trust games. The current study explored propensity to trust, trustworthiness, and trust behaviors in a new computer-mediated trust relevant task. We used multivariate multilevel survival analysis (MMSA) to analyze behaviors across time. Results indicated propensity to trust did not influence trust behaviors. However, trustworthiness perceptions influenced initial trust behaviors and trust behaviors influenced subsequent trustworthiness perceptions. Indeed, behaviors fully mediated the relationship of trustworthiness perceptions over time. The study demonstrated the utility of MMSA and the new trust game, Checkmate, as viable research methods and stimuli for assessing the loci of trust.
\end{abstract}

Keywords Trust $\cdot$ Multilevel $\cdot$ Structural equation modeling · Survival analysis $\cdot$ Social interaction

Researchers have had a longstanding interest in interpersonal decision making, specifically in relationship to making oneself vulnerable to another (i.e., trusting others). Deciding to be vulnerable can have deleterious or advantageous effects (Ferrin, Bligh, \& Kohles, 2007), as one's trust may be taken advantage of or reciprocated. Trust is an important aspect of

Gene M. Alarcon

Gene.alarcon.1@us.af.mil

1 Air Force Research Laboratory, Wright Patterson AFB, 2215 First St. Bldg 33, Dayton, OH 45433-7604, USA

2 Ball Aerospace \& Technologies Corp., Boulder, CO, USA

3 CSRA International Inc., Dayton, OH, USA

4 Consortium of Research Universities, Washington, DC, USA human social behavior. Indeed, interpersonal trust is crucial in developing lasting friendships, a secure social life, and longlasting marriages (Deutsch, 1958; Simpson, 2007). Interpersonal relationships in one's private life also rely on trust (Kramer, 1999; Kreps, 1990; Mayer \& Davis, 1999; Schaubroek, Peng, \& Hannah, 2013). Trust is imperative to establishing relationships among strangers (Foddy, Platow, \& Yamagishi, 2009), such as when they participate in transactions in various virtual and non-virtual marketplaces (e.g., eBay, Craigslist, farmer's market).

The trust process can be divided into three concepts: trusting beliefs (i.e., trustworthiness; Colquitt, Scott, \& LePine, 2007; Mayer, Davis, \& Schoorman, 1995), trusting intentions (i.e., willingness to be vulnerable; Mayer et al., 1995), and trusting actions (e.g., reliance on others; Pillutla, Malhotra, \& Murninghan, 2003). Trust beliefs include both trait and state influences on the trust process such as individual differences in personality (propensity to trust) and state beliefs (trustworthiness). The latter assessment is influenced by the trustor, perceptions of the trustee (based on behavior, verbal interactions, etc.) and the environment. Trust intentions are the willingness to be vulnerable to a referent (e.g., group, person). Trust actions are the behaviors one performs when relying on the referent. It should be noted trust intentions do not perfectly correlate with actions. Multiple constructs may play a role in subsequent behaviors, as has been noted in the social psychology literature (Ajzen, 1991). As such, a trustor may have high trust intentions and not perform a trust behavior. Conversely, a trustor may also have low trust intentions and still perform the behavior. It is important to explicate these three concepts to more accurately assess the trust process.

While models of trust have received considerable attention in recent years, empirical tests of the models are limited in that 
few articles employ analysis of trust actions over time, and those that do tend to assess trust behavior dichotomously, if at all, with the operationalization of trust behavior often restricted to strictly loss or gain (see Wedekind \& Milinski, 1996). The majority of extant longitudinal research has focused on trust intentions, perceptions of trustworthiness, or predictors of the two, rather than trusting behaviors (Colquitt \& Rodell, 2011; Levin, Whitener, \& Cross, 2006; Schaubroek et al., 2013). For instance, Schaubroek and colleagues (2013) assessed cognitive and affective trust over time, assessing self-report trust intentions. Colquitt and Rodell (2011) examined how perceptions of justice and trustworthiness impact trust intentions over time, assessing self-report of trust beliefs. Furthermore, Levin et al. (2006) explored the antecedents of trust over time, assessing self-report of trust beliefs. All three of these studies have explored trust exclusively through selfreport, albeit, over time. This is problematic as trust intentions and trust actions are distinct, albeit related, constructs, and one cannot make strong inferences about the trust process by using trust intentions as the sole outcome. The previous longitudinal studies focused on trust intentions or trust beliefs; as such, it is difficult to determine how trust beliefs, trust intentions, and trust actions are truly related. Thus, not only is there a critical need for additional longitudinal research on the trust process, but research is specifically needed to examine how theoretically-driven trust antecedents (e.g., propensity to trust and trustworthiness perceptions) impact trust behavior over time. The current study builds on the previous research as it encompasses all three aspects (trusting beliefs, trusting intentions, and trusting actions) of the trust process over time. This enables us to more accurately determine how trust intentions and beliefs influence trust behaviors, and how those behaviors shape subsequent beliefs.

\section{Trust}

Trust represents the decision of an individual to accept vulnerabilities associated with another individual, often without the ability to monitor the actions of the other individual (Mayer et al., 1995). Specifically, interpersonal trust involves a sense of vulnerability or risk. Without the decision to be vulnerable or at risk, there is no need for trust. Interpersonal trust is also inherently relational. To be vulnerable, one must be vulnerable 'to' some other individual, such as a spouse, a supervisor, or a cab driver for example. Lastly, interpersonal trust involves positive expectations that obligations will be kept by the trustee, regardless of the trustor's ability to control and/or monitor the actions of the trustee.

There is no dearth of trust theories and models in the literature. However, recent research has suggested a combination of two models, namely the rational choice model (Lewicki \& Bunker, 1995; Shapiro, Sheppard, \&
Cheraskin, 1992) and information processing model (Levin et al., 2006; Lewicki \& Bunker, 1995; Shapiro et al., 1992). These two models have differing loci for the trust decision. The rational choice model states individuals will make logical decisions based on the greatest benefit for themselves. The rational choice model is inherently focused on the trustors, their perceptions, beliefs, and attitudes. With little information in new scenarios, people may rely on the rational choice model (Weber, Malhotra, \& Murninghan, 2004) - where their trust decisions (as the trustor) are based primarily on dispositional influences, desires, or perceptual biases. However, over time, trustors may transition to an information processing approach, whereby information is gained about the trustee and an emphasis is placed on the trustee rather than the trustor (Jones \& Shah, 2016). As more information becomes salient about the trustee, the trustor will process the information and rely on the information to make decisions. There are many different aspects of information the trustor may rely on, but we focus on antecedents found in the trust literature.

\section{Antecedents of trust}

A critical characteristic for understanding trust is the delineation of trust actions from its antecedents (i.e., propensity to trust and trustworthiness; Mayer et al., 1995). The Mayer et al model depicts propensity to trust and trustworthiness as predictors of trust intentions and trust actions. Although these constructs are related, it is important to note they are theoretically distinct. One's propensity to trust is a target-agnostic trait-based perception of others, whereas trustworthiness is an informationbased perception of a particular target. In either case, there exists the potential for inaccuracies; however, the perceptions will influence attitudes and behaviors regardless of their accuracy. Notably trustor influences, such as one's propensity to trust, operate early in the trust process whereas trust beliefs and trust actions impact the trust process in the later stages of an interaction, indicating a transition from a rational choice model to an information processing model (Alarcon, Lyons, \& Christensen, 2016; Jones \& Shah, 2016). In the present paper, we adopt a similar approach and consider trustor effects (trust propensity), trust beliefs (trustworthiness beliefs), and trust actions (trust behavior) over time. We label these effects for propensity to trust, trustworthiness, and trust actions as trustor, trustee, and trust actions, respectively, which is consistent with Jones and Shah (2016).

Propensity to trust An important antecedent of trustworthiness and trust actions involves characteristics of the trustor, namely 
one's propensity to trust. Propensity to trust is an individual difference characteristic that refers to the general tendency for someone to trust other individuals (Mayer et al., 1995; Hochreich \& Rotter, 1970). Propensity to trust has a global effect on trust intentions (Colquitt et al., 2007) and trustworthiness beliefs (Jones \& Shah, 2016). However, the impact of trust propensity is most salient early in interpersonal interactions, when other information may not be available (McKnight, Cummings, \& Chervany, 1998). During such situations, an individual deliberately makes a comparison of the potential costs and potential benefits from a given set of decision options, and then selects the most optimal course of action (Jones \& Shah, 2016) based on the rational choice model. In the absence of other information, the individual may be driven by the only information available, namely the dispositional biases brought to the situation. In terms of trust, in the absence of information about another individual, the decision to trust is likely to depend on an evaluation of the likelihood of incurring a loss versus being rewarded for trusting based on one's dispositional propensity to trust.

Trustworthiness Another important antecedent is trustworthiness, which is the trustor's perception of the trustee (Mayer \& Davis, 1999). Perceived trustworthiness has been conceptualized as a trustor's perception of the trustee's competence, benevolence, and integrity. These perceptions are formed as a trustor interprets and ascribes motives to the trustees' actions (Ferrin \& Dirks, 2003). Thus, perceptions of trustworthiness, although inherently within the trustor, are a function of the interaction of trustor and trustee as the trustor is processing information about the trustee. It is important to note these are the ascribed beliefs of the trustor and are not necessarily factual. Although the perceptions may not be accurate, they infer an informationprocessing model to trust behaviors over time. As the relationship develops, more information becomes known over time and hence, is available to guide trustworthiness beliefs. As interactions mature, the trustor will increasingly depend on the behavior of the trustee rather than personal dispositional factors, such as propensity to trust, when making trust evaluations (Jones \& Shah, 2016; Levin et al., 2006). Furthermore, trust may manifest by risk-taking behaviors unique to that relationship (Dietz \& Fortin, 2007; Dirks, 1999), impacting subsequent trustworthiness beliefs.

Trust actions Trust is founded on a social exchange process (Blau, 1964) whereby actions, if reciprocated, will be met with trust. Trust actions are the risk-taking behaviors indicative of a trusting relationship (Pillutla et al., 2003). Research has demonstrated trust behaviors from one individual beget trustbased behaviors from others, which in turn yield heightened trustworthiness from the other individual (Serva, Fuller, \& Mayer, 2005). An initial trusting behavior has a significant influence on later trusting behaviors within a dyad.
Specifically, as a trustor trusts in the trustee, that trust may be reciprocated by the trustee, which in turn leads to increased trust by the trustor. This is known as reciprocal trust (Serva et al., 2005).

\section{Trust actions and trust games}

The investment/dictator scenario is an economic decisionmaking game (Berg, Dickhaut, \& McCabe, 1995). The game has been used to model trust, as trust relevance requires some stake (i.e., risk), be it social, financial, personal, or organizational in nature (Parkhe \& Miller, 2000). Thus, trust-based scenarios involving real vulnerability are a critical requirement for trust research. The investment/dictator game is a two-person game where person A receives an initial endowment and can keep the money or send some or all of it (depending on the experiment) to the other person in the dyad, person $\mathrm{B}$. If the money is sent to person $\mathrm{B}$, it increases in value by several fold, usually doubling. Person B then decides whether to keep the money or send it back to person A. The rules are typically known to the players in the game, such as how much money there is to start with and how much it will increase if sent to the other player. It is seen as a trust game because the players are vulnerable, giving money to another, and they need to trust the other will send money back. The more money sent, the higher the trust. In the investment/ dictator game the average money sent is about half the endowment and the returns from person B are typically $30-40 \%$ of the money received (see Johnson \& Mislin, 2011). An advantage of the investment/dictator game over other trust scenarios, such as the Prisoner's Dilemma (see Wedekind \& Milinski, 1996), is the possibility of both loss or gain of money depending on their decision. Research has indicated participants behave differently according to the parameters of the game, namely loss or gain (Thaler \& Jonshon, 1990). Specifically, in the presence of prior gain, such as winning money, participants are typically apt to wager more. However, there are still some issues with the trust game.

The investment/dictator game scenario is unlike most naturalistic trusting interactions. First, an integral part of trust is the lack of monitoring or ambiguity in the situation (Mayer et al., 1995). In the investment/dictator game described above, the trustor knows the actions of the trustee, i.e., how much money they sent back out of the full amount. For example, if person A sends person B US\$10 and knows it will be doubled (US\$20), person A knows what amount of money person B is able to send. There is no ambiguity about whether one made the right decision, because if person B sends back US\$6 (30\% of US\$20 as per meta-analysis; Johnson \& Mislin, 2011), person A knows how much to trust person B. This is a common issue with many trust games, in that there is no ambiguity 
as to whether the partner performed a trusting behavior. Second, there is an issue with the task being overly simple. The decision to send the money relies on the benevolence and integrity aspects of trustworthiness; there is no ability component involved, which limits the generalizability of typical trust games to the trust process. Trust is often more complex than a simple decision, as it also involves the trustee having the ability to perform the promised behavior, and ability is a consistent predictor of trust intentions (Serva et al., 2005).

To more closely approximate naturalistic environments and more effectively examine interpersonal phenomena in them, the Checkmate task was created for the current study. The Checkmate task attempts to insert both ambiguity and all three facets of trustworthiness (including ability) into the traditional investment/dictator game so as to explore the full range of the trust process. The Checkmate task is a computer-mediated, trustrelevant social exchange task. The task was developed to assess trust in dyads in which dishonest behaviors are not explicitly known. The trustor ("Banker") sends money to invest in a "Game Player" (GP). Depending how well the GP played the game, the money is increased by a percentage. However, the GP also has the opportunity to lose part of the endowment because of poor performance. This creates a cover for the GP to keep more money (a low return on investment was due to poor performance). In addition, the wagers for the Banker overlap so the Game Player is unsure of the precise level of wager placed on his/her performance (see Method section).

For this task, the Banker is the focal trustor and the GP is the trustee. Specifically, the Banker is the person with high vulnerability. Once the wager is made, the Banker is able to see part of the GP's performance, but performance is inferred versus directly observed as the Banker cannot see the boxes being collected (see Method section). The Banker must assess the benevolence and integrity of the GP as in the investment/ dictator game, but must also determine the perceived ability of the GP. In addition, the Banker is unable to fully monitor the GP, creating greater affordances for trust. The GP is also vulnerable but to a much less degree; if the GP betrays the Banker, the Banker may wager less in the future, protecting his/her endowment and minimizing the risk. This would have an adverse impact on the GP as s/he will not earn larger winnings in the future. In addition, the GP has an idea of the wager placed on him or her (see Method section) but also has uncertainty, as the Banker can only select a wager category (small, medium, or large) rather than a specific amount. A key difference between the Banker and the GP is the Banker's role does not require ability from the GP's perspective of trust, rather the Banker simply determines how much to wager.

Propensity to trust and trust actions As a stable trait, propensity to trust should influence wager behaviors through a cost-benefit analysis. Initial trust perceptions will be influenced by stable personality traits, as other information is unavailable (Lewicki \& Bunker, 1995; Shapiro et al., 1992). In this context, cost-benefit analyses, biases, and predispositions all reside solely within the trustor. Once other information becomes more salient, such as the trustee's previous behaviors, propensity to trust will have a weaker influence on the extent to which the trustor will make him/herself vulnerable to the trustee (Mayer et al., 1995). Indeed, research has consistently found the influence of trustor effects to decline as trustee information becomes available (Alarcon et al., 2016; Jones \& Shah, 2016; McKnight et al., 1998). As such, we expect individuals higher in propensity to trust to differ from individuals lower in propensity to trust in their likelihood of performing a trusting behavior.

\section{- Hypothesis 1: Propensity to trust will be positively related to higher trust behaviors.}

Trustworthiness and trust actions Although propensity to trust should be a strong predictor of initial trust actions, trustworthiness also influences behavior. Trustworthiness perceptions are cognitive and affective assessments of the partner. Although perceptions of trustworthiness should be low in initial conditions, as information is limited (i.e., ability to complete task, integrity, etc.), higher initial perceptions of trustworthiness should be related to wager level. These initial trustworthiness perceptions will be driven by the Banker's propensity to trust. In theory, propensity to trust should be related to behaviors both directly (hypothesis 1 ) and indirectly through higher initial trustworthiness perceptions.

\section{Hypothesis 2: Initial perceptions of trustworthiness will} be positively related to higher trust behaviors.

As more information becomes known throughout an interaction, trustors will progress from a rational choice model to an information processing model (Jones \& Shah, 2016). The transition to an information processing model indicates the trustee's characteristics drive future trustor perceptions of trustworthiness. Indeed, trustee behaviors are indicative of trustworthiness once such information has been observed. These outcomes accumulate and eventually create a feedback loop to influence subsequent perceptions of characteristics of the trustee (i.e., trustworthiness; Axelrod, 1984; Korsgard, Brodt, \& Whitener, 2002; Lewicki \& Bunker, 1995). Thus, we expect trust behaviors will be associated with trustor perceptions. Consistent with the reciprocal nature of trust, we expect trust actions (wagers) will result in higher final trustworthiness perceptions of the GP by the Banker (Serva et al., 2005).

Hypothesis 3: Trust behaviors will be positively associated with final trustworthiness. 


\section{Method}

\section{Participants}

A total of 189 participants ( $60 \%$ male) with a mean age of 31 years were recruited from the general populations of a US Midwestern and a US East Coast city via newspaper and Craigslist ads. Participants were recruited to play a trust game. Participants received monetary compensation of US\$10 per hour in addition to keeping any of their endowment retained in the course of completing the Checkmate task. Participants kept the hourly rate regardless of performance on the task.

\section{Measures}

Propensity to trust We used Mayer, and Davis' (1999) scale to measure participants' propensity to trust. The scale consists of eight items measuring propensity to trust in general. An example item is, "Most experts tell the truth about the limits of their knowledge." Cronbach's alpha for the current study was 0.70 .

Trustworthiness We used a single-item measure of overall trustworthiness. Participants marked their agreement with the item "I believe my partner (the Game Player) is trustworthy" on a Likert scale of 1 to 7, with 1 indicating "Strongly Disagree" and 7 indicating "Strongly Agree." Although we measured trust with one item, single-item measures have been used in prior trust research (Ferrin, Dirks, \& Shah, 2006; Jones \& Shah, 2016) and such measures are appropriate when multiple item measures are likely to cause response fatigue and when the item is not ambiguous (Sackett \& Larson, 1990; Wanous, Reichers, \& Hudy, 1997).

\section{Experiment platform}

Checkmate The Checkmate task was created for the purposes of the current study, based on the investment/dictator game. The Checkmate task is a computer-mediated task in which participants played either the "Banker" or the "Game Player." The Banker's role was to loan money to the Game Player (GP) out of an initial endowment provided by the experimenters and tracked via software displayed in the upper right-hand corner of the screen; during play with each partner, the Banker began his/her rounds with a US\$50 endowment. The GP's role was to complete virtual mazes, which varied in difficulty. The mazes were three-dimensional worlds featuring small cash boxes and larger impassable obstacles. The GP was instructed to navigate the maze in order to collect as many cash boxes as possible in the time allotted. The number of cash boxes collected (performance) by the GP directly controlled earnings. In the Checkmate task there were three risk levels: Low 75-150\%, Moderate 50-200\%, and
High 0-300\%. For example, in the Low-risk maze, the GP could lose up to $25 \%$ of the loan for a poor performance, leaving the GP with $75 \%$ of the loaned amount. A good performance could earn up to $50 \%$ interest on the loan leaving the GP with $150 \%$ of the loaned amount. Moderate and High risk followed similar payoff structures with the appropriate payoff percentages as shown above.

Poor performance resulted in a loss on that loan, while high performance resulted in additional earnings per the payout matrices described above. All money earned and lost in the course of game play was real; earnings from the Banker's loan as well as anything remaining from the initial endowments were added to the GP's compensation based on the choices he/she made during each round. The GP then decided how much of the compensation to send back to the Banker. A single round of the Checkmate task is detailed next in the Procedure.

\section{Procedure}

Participants arrived at the laboratory at 8:30 a.m. Participants completed a comprehensive written informed consent. Participants then viewed an informational Powerpoint about the task. The Powerpoint described how to perform the task, illustrated the process of playing the game (as both the Banker and GP), and explained the compensation. After the slideshow participants filled out background surveys, including propensity to trust. Background surveys were filled out at the beginning of the day only once. After completing the background surveys and a short practice session, participants then briefly met in person with their first partner (no longer than five minutes). Participants were informed they would play five rounds in the role of the Banker and five rounds in the role of GP with each partner (for a total of 20 rounds). Each set of five rounds was considered a "block," and each set of two blocks was considered a "session." Overall, individuals participated in two sessions, comprised of ten rounds each, with different partners for each session. Figure 1 illustrates the participants' roles during a typical testing day. The procedure for each round was as follows:

1) The GP made a promise to the banker to run the maze at a specific risk level (High, Medium, or Low).

2) The GP promised a return selection from one of the following:

a. Return initial loan and ALL gains to the Banker.

b. Return initial loan and $50 \%$ of the gains to the Banker.

c. Return $50 \%$ of the money available at the end of the round to the Banker. 


\begin{tabular}{|c|c|c|c|}
\hline Session 1 & Participant & $\begin{array}{c}\text { Block 1 } \\
\text { Rounds 1-5 }\end{array}$ & $\begin{array}{c}\text { Block 2 } \\
\text { Rounds 6-10 }\end{array}$ \\
\hline Partners & $\begin{array}{l}\text { Participant 1's } \\
\text { Role: } \\
\text { Participant 2's } \\
\text { Role: } \\
\end{array}$ & $\begin{array}{c}\text { Banker } \\
\text { Game Player }\end{array}$ & $\begin{array}{c}\text { Game Player } \\
\text { Banker }\end{array}$ \\
\hline Partners & $\begin{array}{l}\text { Participant 3's } \\
\text { Role: } \\
\text { Participant 4's } \\
\text { Role: } \\
\end{array}$ & $\begin{array}{c}\text { Game Player } \\
\text { Banker } \\
\end{array}$ & $\begin{array}{c}\text { Banker } \\
\text { Game Player }\end{array}$ \\
\hline Session 2 & Participant & $\begin{array}{c}\text { Block 1 } \\
\text { Rounds 1-5 } \\
\end{array}$ & $\begin{array}{c}\text { Block 2 } \\
\text { Rounds 6-10 }\end{array}$ \\
\hline Partners & $\begin{array}{l}\text { Participant 1's } \\
\text { Role: } \\
\text { Participant 3's } \\
\text { Role: }\end{array}$ & $\begin{array}{c}\text { Banker } \\
\text { Game Player }\end{array}$ & $\begin{array}{c}\text { Game Player } \\
\text { Banker }\end{array}$ \\
\hline Partners & $\begin{array}{l}\text { Participant 2's } \\
\text { Role: } \\
\text { Participant 4's } \\
\text { Role: }\end{array}$ & $\begin{array}{c}\text { Game Player } \\
\text { Banker }\end{array}$ & $\begin{array}{c}\text { Banker } \\
\text { Game Player }\end{array}$ \\
\hline
\end{tabular}

Fig. 1 The roles for each participant throughout a typical day of testing

d. Return an amount not listed here to the Banker.

e. I will decide how much money to return to the Banker at the end of the round.

3) Banker was informed of GP's promises (Steps 1 and 2) through Checkmate software.

4) Banker selected actual loan amount via three categories (Banker was not in control of actual amount sent within the category; actual amount depended on the round, the first round each wager was US\$1 less than the overlapping section of the wagers, i.e., in round 1 a small wager was US $\$ 3$, the wager amount increased by US $\$ 1$ for each round, i.e., a small wager in round 2 was US\$4):

a. Small (\$1-\$7).

b. Medium (\$4-\$10).

c. Large (\$7-\$13).

5) GP received Banker's money (but not actual loan category).

6) GP chose actual risk level (Note: This choice does not have to be the same as what the GP promised the Banker and the Banker cannot see the selection).

7) The GP played the actual maze for $2 \mathrm{~min}$. The actual maze risk selection makes it possible for the GP to take larger risks with larger potential earnings, enabling the GP to retain more earnings undetected.

8) While the GP completed the maze, the Banker was given a top-down view of the maze map with the ability to watch the GP's progress through the maze. However, this view was designed to give a vague impression of the progress. The Banker could not see the location of cash boxes or any other information about the GP's performance; thus, the actual risk level of the maze was masked to the Banker.

9) After completing the maze, the GP learned how the performance affected the loan and could split the loan and earnings with the following options (Note: The GP was not obligated to honor the original promise):

a. More than promised.

b. Exact amount promised.

c. Less than promised.

d. Other amount.

10) The Banker was informed via computer of the amount of money the GP returned that round. No other information was given (i.e., difficulty chosen, actual amount earned, etc.).

11) Banker completed the post-round questionnaire, including the trustworthiness item.

These 11 steps comprised a single round. After the first session of the day (five rounds as the banker and five rounds as the game player) participants were provided with a break and snacks. After their break, participants switched partners, performing a new session of ten rounds (five as banker and five as game player). After all 20 rounds (two sessions), 
participants were compensated for their participation at an hourly rate and given their earnings from the task. The total study ran approximately 5 hours.

\section{Coding behavioral data}

We coded the behavioral data from the GP in the Checkmate task into three categories: (1) honest, (2) dishonest, and (3) inability. Honest was coded as the GP splitting the loan and earnings exactly as promised or more. For example, if the GP promised $50 \%$ of the earnings from the round and returned 50 $\%$ of the earnings or more, it was coded as an honest behavior. If the GP promised $50 \%$ of the earnings from the round and returned less than $50 \%$ of the earnings, the behavior was coded as dishonest. Lastly, if the GP was unable to return the amount promised because of poor performance in the task, therefore having less than the initial wager by the banker, the behavior was coded as inability. We coded this behavior as different from distrust because the GP's behavioral intent may have been to return the promised amount, but was unable to return the amount due to lack of funds. Banker's behavioral data was already classified into categorical data when they chose their wager category, High, Medium, or Low. This created dyadic data points given the behaviors of the pair (see Table 1 for all possible combinations).

\section{Multivariate multilevel survival analysis of dyads}

New advances in statistical analyses may provide better avenues for analyzing and understanding behavior in dyads. The multivariate multilevel survival analysis (MMSA; Stoolmiller \& Snyder, 2006, 2014) framework is an advanced statistical technique that allows researchers to answer questions about timing of events in dyads. ${ }^{1}$ The MMSA framework grew out of the survival analysis literature; survival analysis is the study of time durations until one or more events occur (see Tabachnik \& Fidell, 2013, for an introduction). Survival analysis seeks to determine how long until one leaves a condition, thus time in survival analysis focuses on duration in a condition. The analyses seek to predict the hazard rate - or waiting time until an event occurs - using log odds. Higher hazard rates indicate a shorter waiting time until the event occurs. In contrast, lower hazard rates indicate a longer waiting time until the event occurs. Hazard rates can change over time due to observed or unobserved influences. Lastly, censoring is an important aspect of the survival analysis model. Censoring is when the observation period ends but the event did not occur for some participants. Censoring in survival analysis takes into account all known information about the waiting time for censored participants; in other words, the

\footnotetext{
${ }^{1}$ For readers interested in an in-depth discussion of MMSA, we refer them to Stoolmiller and Snyder (2006).
}

Table 1 All possible dyadic interactions and the frequency of the interactions

\begin{tabular}{lllll}
\hline Game Player's behavior & \multicolumn{2}{l}{ Banker's wager } & \multirow{2}{*}{ Total } \\
\cline { 2 - 4 } & Low & Medium & High & \\
\hline Honest & LH (450) & MH (186) & HH (138) & 774 \\
Dishonest & LD (137) & MD (65) & HD (31) & 233 \\
Inability & LIA (46) & MIA (31) & HIA (33) & 110 \\
Total & 633 & 282 & 202 & 1117 \\
\hline
\end{tabular}

Note: Dyadic behavior is represented by the first letter of the Banker's wager and the second letter of the Game Player's behavior; numbers in parentheses indicate number of times dyads were in that state

waiting time is not known but it is at least as long as the observation period.

Multilevel analyses have increasing utility in a variety of linear and non-linear models (Hox, 2010; Tabachnik \& Fidell, 2013). Dyads are small groups with an outcome dependent on the combined behavior, and thus they are multilevel. Multilevel analyses have also been applied to survival analysis successfully (Stoolmiller \& Snyder, 2006, 2014). Research has demonstrated the capability to predict the probability of a partner continuing a behavior (survival analysis) in a dyad (multilevel). Typically in survival analysis, the event of interest is treated as the end point, for example death. However, current research with the MMSA framework attempts to utilize hazard rates as predictors, outcomes, and correlates of other behaviors, making the model multivariate. For example, Stoolmiller and Snyder (2014) explored length of time spent in a negative or positive emotional state as a predictor of future antisocial behavior. This methodology can be extended to analyzing trust in dyads. Participants perceiving their partner as trustworthy will engage in more trust behaviors than in a relationship where the partner is perceived as not trustworthy (i.e., Hypotheses 1 and 3 above). Time in the current study is length of time a dyad spends in a condition, as either can leave the condition.

The MMSA framework builds upon the Cox model of survival analysis. The Cox model is utilized because the base hazards do not need to be specified (i.e., the model is semiparametric), which eliminates chances of misspecification because it is one less parameter that needs to be constrained (Stoolmiller \& Snyder, 2014; Therneau \& Grambsch, 2000). Figure 2 represents a path diagram of the MMSA framework (see Appendix A for MMSA equation). As is the case with structural equation modeling, latent variables are depicted with circles, observed variables are depicted with squares/rectangles, and regressions are depicted with straight arrows. Multilevel aspects of the model are depicted with a horizontal line differentiating between within-dyads and between-dyads. As survival analysis is non-linear, the indicators between the 
$\log$ hazard rates and the baseline hazard function to the episode durations are depicted with crooked arrows.

In MMSA analyses there are two sets of behaviors that influence the dyad, the Banker and the GP. Each partner is coded with three options. The Banker can perform a High, Medium, or Low wager, indicating their level of trust in the GP. The GP may perform an Honest, Dishonest, or Inability to be honest action. Table 1 illustrates all possible behavioral outcomes for the dyads. Thus, either partner can terminate the condition by changing wager habits (banker) or by performing honest behaviors (GP). The MMSA model depicted in Fig. 2 illustrates a dyadic interaction given a Banker in the Medium (M) wager condition and the GP has performed an honest $(\mathrm{H})$ behavior, abbreviated as $\mathrm{MH}$. To simplify the model for analyses, one partner's behavior is fixed across the model, in this instance the honesty of the GP's behavior. The path diagram illustrates the hazard rate, given the honesty of the GP's behavior and the Banker's wager $(\mathrm{MH})$. The hazard rate is required to be non-negative to make sense. The model involves two pieces: the baseline hazard function $\mathrm{h}_{\mathrm{OMH}}(\mathrm{t})$ and the exponential term that includes all the predictors, latent or observed. The baseline hazard function allows the hazard to function over time but not across individuals. The exponential term does allow the hazard to vary across individuals. The latent variable in Fig. 2 represents individual differences on the log hazard scale, which is important to remember when interpreting effect sizes. The observed variables that define the latent hazard rates represent both duration of $\mathrm{MH}$ - indicating the initial state of $\mathrm{MH}$ and the terminating event - and a dichotomous censoring indicator within the single square box. It is represented this way because, although they are separate variables, they must go together to avoid biased estimates of the hazard rates. The observed duration of behaviors within each dyad (in the current example, Banker's behavior of M and GP's behavior of $\mathrm{H})$ are the level 1 variables, as are the censor indicators, similar to a latent growth model. The between-dyad differences (Banker's perception of GP's trustworthiness) are predictors of the latent variable of dyadic behavior.

The difference between a standard regression intercept and the baseline hazard function is that the baseline hazard function is a function of time; in the current study, time is the duration in a given behavior condition for both partners. This indicates the population rate of event occurrence can change over time while waiting for the event to happen; this can be the independent of predictor in the model. In the current context, the hazard of a Banker's medium wager and an honest behavior by the GP (MH) may change over time, independent of variables assessed in the current study. Additionally, the final model has multiple hazard rates to which a participant can change, making the model multivariate.

Lastly, censoring is modeled differently in the MMSA framework than in typical survival analysis. The Banker can leave a state of Medium wager for multiple competing potential end states $(\mathrm{HH}, \mathrm{MH}$, or $\mathrm{LH})$, and the occurrence of one precludes the occurrence of all others. The Banker choosing to change from a Medium wager state to a High wager state indicates the Low wager state was censored, as it did not occur in the transition state, but there was a probability it could have occurred. The advantage of the MMSA model is that we can add predictors of the latent hazard rates. In the current study, we can predict the latent hazard rates with propensity to trust and perceptions of trustworthiness at time point 1 . In addition, we can use the latent hazard rates to predict variables. In the

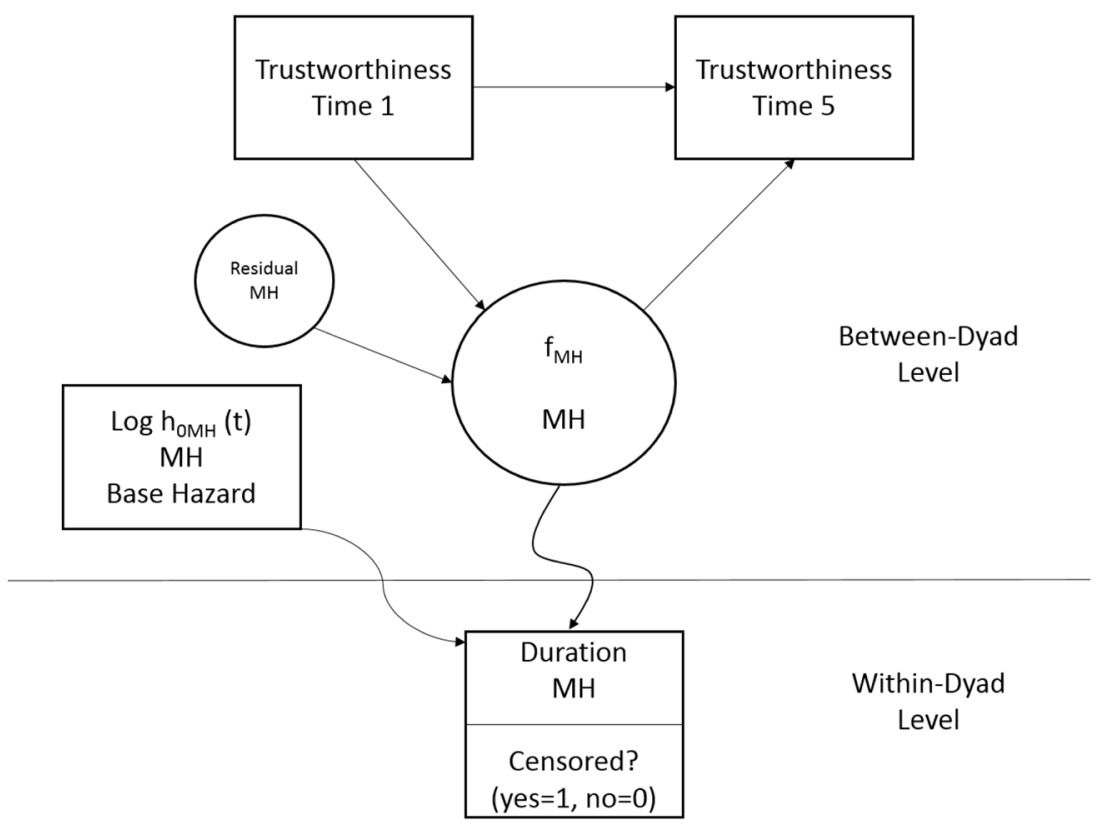

Fig. 2 Heuristic path diagram of the multilevel Cox model for hazard rate of Banker high wager 
current study, we sought to predict final perceptions of GP trustworthiness (time point 5).

In summary, the employed analyses explored the duration a dyad stayed in a behavioral condition (HH or $\mathrm{MH}$ ) using survival analysis. We used the latent variables of duration as outcomes and predictors of trustworthiness perceptions. Thus, we were able to explore if self-reports of propensity to trust and trustworthiness predict behavior and if those behaviors predict subsequent self-reports of trustworthiness.

\section{Results}

Propensity to trust had a mean of 2.79 with a standard deviation (SD) of 0.53 . The range of the scale was $1.50-4.25$. The mean level of propensity to trust was lower than found in previous research on students and middle management (Gill, Boies, Finegan, \& McNally, 2005; Jarvenpaa, Knoll, \& Leidner, 1998). However, the mean was similar to other research that has explored propensity to trust among the general public (Alarcon et al., 2015). Trustworthiness at Time 1 had a mean of 5.41 and a SD of 1.34. Trustworthiness at Time 5 had a mean of 5.33 and a SD of 1.59. The ranges for both time points were 1-7. Trustworthiness at Time 1 was related to Time $5(r=.669, p<.001)$. Behaviors will be abbreviated by the conditions that they represent; Banker's trusting actions will be represented with $\mathrm{L}$ (low), $\mathrm{M}$ (medium), and $\mathrm{H}$ (high) wagers. The GP's behaviors will be represented by $\mathrm{H}$ (honest), $\mathrm{D}$ (dishonest), or I (inability). The Akaike Information Criterion (AIC) and Bayesian Information Criterion (BIC) were used to determine model fit. The AIC and BIC do not test a model in comparison to a null hypothesis (Kline, 2005). Indices such as the chi-square fit index and other typical SEM indices provide test against a null model based on means, variances, and covariances (Kline, 2005). Not all of the variables in the MMSA model have these. As such, the AIC and BIC are used to determine relative fit between competing models, illustrating which one fits the data best out of the competing models.

One potential concern with the study design was any carryover effects from participants starting the interaction as the banker or GP first. To determine if any carryover effects were present, we conducted two three-way contingency tables consisting of session number, round number, and behavior to test for proportional independence of the banker's betting behaviors and the GP's return behaviors between sessions one and two. A chi-square test of mutual independence failed to provide evidence for dependence between the factors for both the banker's behavior $\chi^{2}(37)=35.57, p=.536$, and the GP's behaviors $\chi^{2}(37)=48.12, p=.104$. This indicates behaviors for both were not significantly affected by the session.

Frequencies of the interactions are displayed in Table 1. The most frequent interactions were interactions in which the GP performed an honest behavior LH (40.3\%), MH $(16.7 \%)$, and $\mathrm{HH}(12.4 \%)$. Of interest is that the low wagers' behavior was the most frequent, followed by medium and high wagers. Similarly, wagers, given a dishonest behavior, followed the same pattern: $\operatorname{LD}(12.3 \%), \mathrm{MD}(5.8 \%)$, and $\mathrm{HD}$ $(2.8 \%)$. Lastly, wagers given a GP's inability to send back the promised amount followed a similar pattern LI (4.1\%), MI $(2.8 \%)$, and HI $(3 \%)$. The change states of the GP for Dishonest and Inability were not modeled in the current study, as they were rare. Including them in the model as a possible alternative would greatly complicate the model and add little information.

We used MPlus 7.2 (Muthen \& Muthen, 1998-2011) to perform all MMSA analyses. To fit the final model with all three latent hazards, smaller models with one latent hazard and the predictors and outcomes were computed (see Method section; for a full discussion see Stoolmiller \& Snyder, 2014). After completing the single latent factor models, the estimates were used as starting values to fit the final model with all three latent variables. Attempting to fit the finals models without using starting values would result in nonconvergence of the model as they are so complex. The final model resulted in 300 dyad clusters, with a maximum of five level 1 units in each cluster. We tested the ICC to determine if multilevel analyses were appropriate. An ICC above .10 is considered adequate for multilevel analyses (Hox, 2002). The ICC for the outcome trustworthiness at Time 5 was .456 , indicating multilevel analyses are appropriate.

After fitting the Cox model to the data, we were able to plot the baseline hazards by computing the cumulative hazard curve. The cumulative hazard curve illustrates the expected cumulative number of transitions in the interval from 0 to any particular value of time for a hypothetical participant who scores 0 on the latent log hazard variable. The three curves for the Banker's behavior, given the GP is performing an honest behavior, are shown in Fig. 3. Note that duration is on the x-axis, indicating the length of time the dyad spent in the condition. The cumulative hazards are the hazard rate of the dyad leaving the condition over time. The cumulative hazard rates increase over time as the longer the duration in the condition, the more likely it is to leave the condition as the hazard rates are additive. The most salient aspect of the figure is that $\mathrm{LH}$ transitions accumulate much quicker than $\mathrm{HH}$ or $\mathrm{MH}$ transitions. Specifically, by the second trial, $0.4 \mathrm{LH}$ transitions have accumulated, in contrast to approximately 0.15 and 0.05 for $\mathrm{MH}$ and $\mathrm{HH}$, respectively. The LH curve increases as time passes, as we would expect. Dyads where the banker wagered low and the GP performed an honest behavior would want to maximize their profits in the game by increasing their bets. In contrast, dyads where the Banker wagered high or medium and the GP performed an honest behavior appear to have little change in the hazard rate. 
Dyads that wager high and experience an honest behavior will not change their wager as they are profiting. Interestingly, dyads in the medium wager and honest behavior condition did not have a high probability of leaving that condition. This may be due to aspects not assessed in the current study such as risk aversion or some other factors.

We utilized the single hazard models to obtain initial estimates for inclusion in the cumulative model with three hazard rates (i.e., $\mathrm{HH}, \mathrm{MH}$, and $\mathrm{LH}$ ). We conducted two cumulative models: (1) propensity to trust predicting Banker's decision given an honest behavior and (2) trustworthiness predicting Banker's decision given an honest behavior, with the behaviors then predicting final trustworthiness. These will be discussed, each in turn, next.

To determine model fit we ran two models, one with propensity to trust predicting the latent variables and one with propensity to trust covarying with the latent hazard variables. The model for propensity to trust predicting the latent hazards (AIC $=4, \mathrm{BIC}=4,190.300)$ fit the model worse than with propensity to trust covarying with the latent $\log$ hazards $(\mathrm{AIC}=4,140.310, \mathrm{BIC}=4,170.420)$, results are illustrated in Table 2 . The first step was to analyze the variances of the log hazards. As illustrated in Table 2, all of the log hazards, given an honest behavior, had significant variance. These results indicate there are sufficient individual differences to predict the log hazards and to use them as predictors of future outcomes. Contrary to our hypothesis, propensity to trust had no relationship with any of the log hazards as illustrated in Table 1. Hypothesis 1 was not supported. These results indicate participants higher on propensity to trust did not differ from participants low on propensity to trust in their likelihood of a high, medium, or low wager.

Next, we discuss the results of the trustworthiness models. To determine model fit we ran two models, one with trustworthiness predicting the latent variables and one with trustworthiness correlated at each time point but not predicting the latent hazard variables. The model with initial trustworthiness predicting the latent hazards and the latent hazards predicting final trustworthiness had adequate model fit ( $\mathrm{AIC}=$ 4,138.012, $\mathrm{BIC}=4,183.177$ ) and fit the model better than trustworthiness not predicting the latent hazard variables, but instead covarying with the hazards $(\mathrm{AIC}=4,150.307, \mathrm{BIC}=$ 4,170.417). The effects of trustworthiness perceptions on the latent hazards, given an honest behavior by the GP, are illustrated in Table 3. It is important to note, in the initial models with one latent factor per analysis, the relationship between $\mathrm{HH}$ and trustworthiness at time 1 was 0 , and between $\mathrm{HH}$ and trustworthiness at time 5 was 0 . As such, these paths were excluded from the model to ensure convergence. The effects of trustworthiness at time 1 were significantly related to $\mathrm{MH}$ and LH. Results indicate if the Banker perceives the GP as trustworthy, the dyad is less likely to leave the MH condition

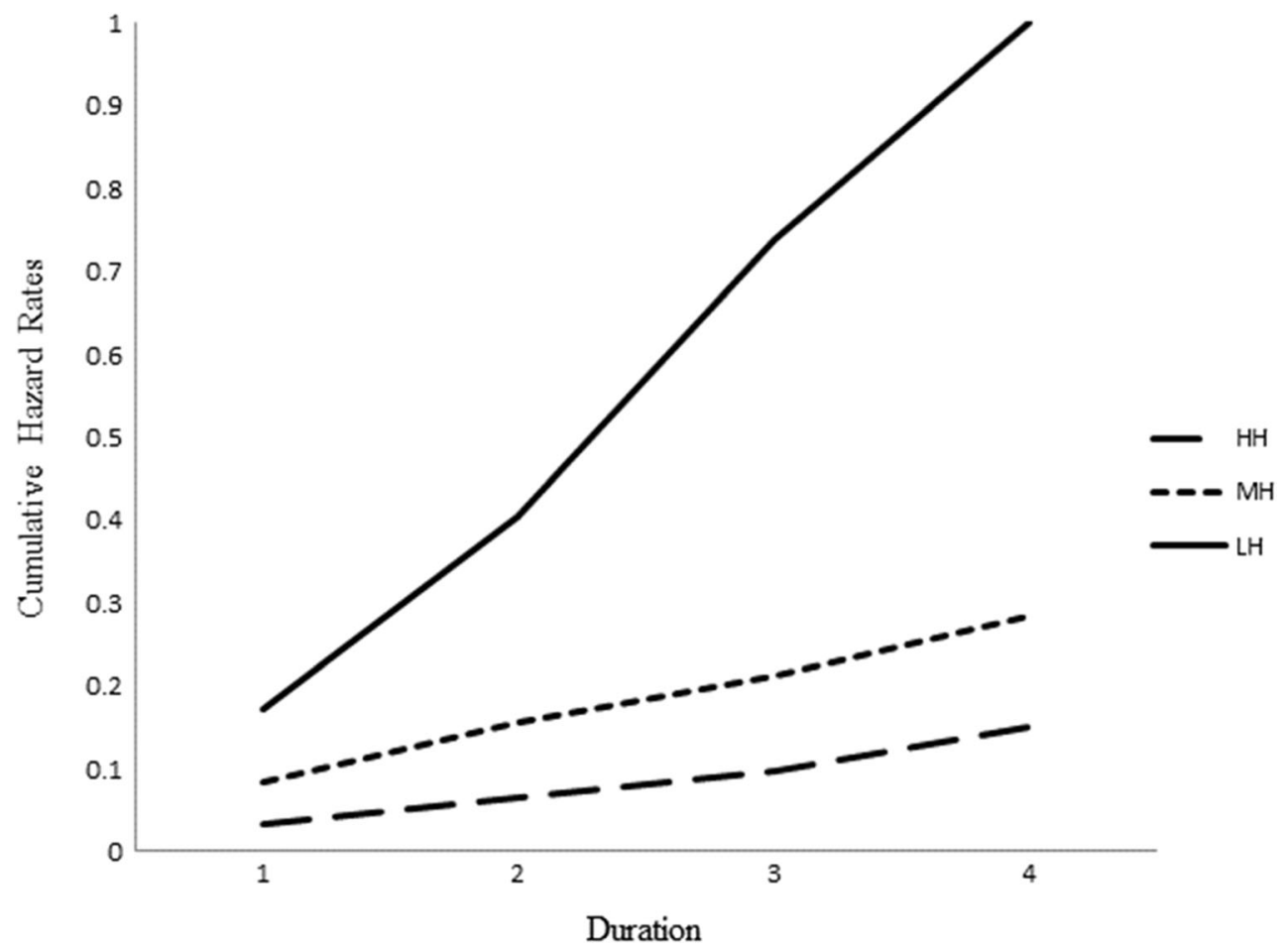

Fig. 3 Cumulative hazard curves for banker hazard rates of High, Medium, and Low Wagers given Game Player trust. Note: Duration on the $\mathrm{x}$-axis indicates how many sessions the dyad stayed in the state. If a dyad stayed in the state over the course of interaction 1 and interaction 2, the duration would be 2 . If the dyad then transitioned to another state, that duration would start at 1 
Table 2 Parameter Estimates, standard errors, critical ratios, and $\mathrm{p}$ values for MMSA for Banker propensity to trust given Game Player honest behavior

\begin{tabular}{lllll}
\hline Effect & Estimate & SE & Estimate/ SE & $p$ \\
\hline Standardized Effects & & & & \\
$\quad$ Propensity to & & & & \\
$\quad$ High-Honest log hazard & 0.079 & 0.149 & 0.532 & .595 \\
$\quad$ Medium-Honest log hazard & 0.056 & 0.115 & 0.486 & .627 \\
$\quad$ Low-Honest log hazard & -0.059 & 0.041 & -1.432 & .152 \\
Correlations & & & & \\
$\quad$ High-Honest log hazard with & & & & \\
$\quad$ Medium-Honest log hazard & 0.525 & 0.196 & 2.679 & .007 \\
$\quad$ Low-Honest log hazard & -0.551 & 0.093 & -5.898 & .000 \\
$\quad$ Medium-Honest log hazard with & & & & \\
$\quad$ Low-Honest log hazard & -0.206 & 0.059 & -3.518 & .000 \\
Variances & & & & \\
$\quad$ High-Honest log hazard & 2.153 & 0.408 & 5.237 & .000 \\
$\quad \begin{array}{l}\text { Medium-Honest log hazard } \\
\text { Low-Honest log hazard }\end{array}$ & 0.708 & 0.172 & 4.115 & .000 \\
\hline
\end{tabular}

Note: SE standard error, $p$ p values

$(-0.14, p<.05)$. In contrast, if the Banker perceives the GP as trustworthy, the dyad is more likely to leave the LH $(0.078, p$ $<.05)$ condition. Thus, hypothesis 2 was partially supported.

Table 3 Parameter estimates, standard errors, critical ratios, and $\mathrm{p}$ values for MMSA for trustworthiness given Game Player trust behavior

\begin{tabular}{lllll}
\hline Effect & Estimate & SE & Estimate/ SE & $p$ \\
\hline Standardized Effects & & & & \\
$\quad$ Trustworthy T1 to & & & & \\
$\quad$ Medium-Honest log hazard & -0.140 & 0.059 & -2.359 & .018 \\
$\quad$ Low-Honest log hazard & 0.078 & 0.036 & 2.192 & .028 \\
Trustworthy T5 on & & & & \\
$\quad$ Trustworthy T1 & 0.699 & 1.951 & 0.358 & .720 \\
$\quad$ High-Honest log hazard & -0.225 & 0.107 & -2.103 & .035 \\
$\quad$ Low-Honest log hazard & 0.767 & 0.416 & -1.846 & .065 \\
Correlations & & & & \\
$\quad$ High-Honest log hazard with & & & & \\
$\quad$ Medium-Honest log hazard & 0.510 & 0.194 & 2.627 & .009 \\
$\quad$ Low-Honest log hazard & -0.543 & 0.094 & -5.769 & .000 \\
Medium-Honest log hazard with & & & & \\
$\quad$ Low-Honest log hazard & 0.189 & 0.056 & -3.395 & .001 \\
Variances & & & & .000 \\
$\quad$ High-Honest log hazard & 2.153 & 0.409 & 5.259 & .000 \\
Medium-Honest log hazard & 0.668 & 0.169 & 3.941 & .000 \\
Low-Honest log hazard & 0.143 & 0.047 & 3.082 & .002 \\
Intercepts & & & & \\
Trustworthy T5 & 5.294 & 0.097 & 54.567 & .000 \\
\hline
\end{tabular}

Note: $S E$ standard error, $p \mathrm{p}$ values, $T 1$ round $1, T 5$ round 5
Next, we used the log hazards to predict final Banker assessments of trustworthiness in the GP. Final assessments of trustworthiness were predicted by longer durations in $\mathrm{HH}$ ($0.225, p<.05$ ). Additionally, although marginally significant, longer durations in the $\mathrm{LH}(0.767, p=.065)$ condition indicated lower final perceptions of trustworthiness. Hypothesis 3 was partially supported. Lastly, in the final models, trustworthiness at time 1 did not predict trustworthiness at time 5 $(0.699, p=.70)$, indicating behaviors fully mediated the relationship.

\section{Discussion}

The trust process is highly complex and recent research has isolated the loci of trustor, trustee, and dyadic influences (Jones \& Shah, 2016). These loci were examined longitudinally in the context of trustworthiness beliefs in Jones and Shah (2015), yet research was needed to study trust behaviors over time. The use of MMSA analyses allowed the trust beliefs and trust behaviors, to be simultaneously assessed. The statistical methodology has clear implications for dyad research in the future. The current study applied a similar structure (i.e., trustor, trustee, and dyad) of trust influences in an exploration of trust behaviors over time. Furthermore, the current study applied a non-dichotomous operationalization of trust behaviors, more closely approximating trust which extends prior research on trust behaviors. This study also included ambiguity to the trust situation, which is a key element for trust to occur (Mayer et al., 1995). Trustor effects (i.e., propensity to trust) appear to have no relationship with trust behaviors in the current task scenario. In contrast, trustee effects (i.e., perceived trustworthiness of the partner) shaped participants' trust behavior. Similarly, in support of the dyadic approach, trust behaviors predicted subsequent trustworthiness perceptions. These behaviors fully mediated the relationship between initial perceptions of trustworthiness and final perceptions of trustworthiness, confirming the dyadic nature of the trustworthiness construct.

\section{Trust behaviors}

The most commonly performed wager by the Bankers in the current study was a Low wager, despite the GP's behavior. Regardless of whether the GP exhibited an honest behavior or not, the Bankers were prone toward conservative investments. This is in contrast to previous research that found investors typically invest $50 \%$ of their endowment in the original investment/dictator game (Johnson \& Mislin, 2011). There are three points of note. First, with the exception of a brief introductory period, the participants in the current study were strangers and had little basis for trust, at least initially. Trust develops over interactions with the trustee; these 
interactions should influence behaviors and perceptions over time (Serva et al., 2005). However, in the investment/dictator game participants are typically strangers as well (Johnson \& Mislin, 2011). Indeed, this development of trust over time can be seen in how initial trustworthiness perceptions were not related to high wager log hazards. Conversely, the $\mathrm{HH} \log$ hazards were significantly related to final trustworthiness perceptions. Participants that spent longer time in the HH condition viewed the GP as more trustworthy, because the GP was performing honest behaviors. Secondly, the likelihood of changing from a conservative wagering strategy to a more aggressive one was more prominent the longer partners interacted when the GP was performing honest behaviors. As such, the participants were clearly willing to accept greater risk as the task progressed. Third, the current study included ambiguity in the performance and intentions of the GP. The inability to monitor the GP, leading to ambiguity of the GP's behavior, may account for the differences between the Checkmate task and the traditional investor/dictator game.

\section{Trustor influences}

Propensity to trust was not related to trust behavior in the current study. Propensity to trust may be an important predictor of trust intentions, but not trust actions. Indeed, propensity to trust has been related to self-report of trust intentions (Colquitt et al., 2007) and trust beliefs (i.e., trustworthiness; Jones \& Shah, 2016). The current results, while surprising, are consistent with research showing that one's propensity to trust has demonstrated weaker correlations with trust intentions than trustworthiness has with trust intentions in the literature and propensity to trust was not directly related to trust actions such as risk taking (Colquitt et al., 2007). However, trust intentions and trust actions are two separate constructs, as behavior is influenced by many psychological aspects (Ajzen, 1991). There are comparatively few studies that have examined the combined effects of trustworthiness and trust propensity on trust behaviors. One may have a high propensity to trust in others across situations, and may perceive trust in others, but environmental aspects such as imminent risk or other psychological variables may also influence the actual trusting behavior. Indeed, Jones and Shah (2016) found trustor effects to diminish over time. Their study involved students in a class project, where the risk outcome, final grade in the course, may not have become an adequate perception of risk until later in the course. In contrast, the risk was prominent for the current participants, as their trust behavior related to risk of monetary loss or gain in the immediate future. The behavior in a complex situation such as Checkmate may rely on dynamic information about the situation, such as imminent risk versus tasks where the consequences for risk occur much later in time. It is also possible that the impact of one's trust propensity on trust behavior is moderated by contextual factors that shape the type of vulnerability. Little research has explored the propensity to trust and its impact on the trust process is not fully understood. Future research should explore the impact of trust propensity under various contextual boundaries.

\section{Trustee influences}

Initial and final trustworthiness perceptions were related to trusting behaviors in the current study, where behaviors fully mediated the relationship between initial and final perceptions. These findings, although seemingly intuitive, are important as the current study is the first to test all three loci of trust and find the proposed mediating effects hypothesized by Mayer et al. (1995) and Jones and Shah (2016). As Bankers gained more information about the GP, they were able to make a more informed decision about their potential future behavior. Given the cue of an honest behavior, the Banker was more likely to leave the Low wager condition to maximize gains and less likely to leave the Medium wager condition. This signals a movement toward an information processing model of the trust process as one considers the trustee influence (Jones \& Shah, 2016).

Initial perceptions of trustworthiness did not influence $\mathrm{HH}$ conditions. This may be accounted for by aspects of the rational choice model. The model dictates a participant may choose the wager condition based on individual differences, perhaps some not measured in the current study. For example, aversion to risk may predict these behaviors. Those high in aversion to risk would choose the Low wager condition as no other information is available (i.e., GP trustworthiness). In contrast, a participant with low aversion to risk may choose the High wager condition when no other information is available. In this situation, the Banker may reason that it is optimal to gain the most amount of money, regardless his perception of the GP, since the GP is performing an honest behavior, thus making longer durations in the $\mathrm{HH}$ condition feasible.

\section{Dyadic influences}

Information processing approaches suggest trustors will base trustee trustworthiness observations on experience with the trustee (Mayer et al., 1995; McKnight et al., 1998), as illustrated in our study. The current study moves beyond prior trust studies by demonstrating the reciprocal nature of the trust process (Serva et al., 2005). In our study, trustworthiness perceptions led to trusting behaviors, which in turn, fostered higher trustworthiness. In fact, trusting behaviors fully mediated the relation between initial and final trustworthiness. In addition, those relationships were different over time in that initial trustworthiness was not related to $\mathrm{HH}$ but $\mathrm{HH}$ significantly predicted final trustworthiness perceptions. These findings extend the trust literature in two important ways. First, the 
results demonstrate that longitudinal research is essential for examining the dynamics of dyadic effects (Jones \& Shah, 2016). Second, these results further confirm the reciprocal linkages specified by Mayer et al. (1995) and supported by Mayer and colleagues (2005). Third, the differences from the traditional investor/dictator game indicate ambiguity played a role in the decision-making process; the inability to monitor the GP may account for the differences between the Checkmate task and the traditional investor/dictator game wagering percentages. However, this may also be accounted for by several other factors such as the computer-mediated aspect of the task. Fourth, trustee's behavior, through the dyadic interactions, influenced trustworthiness perceptions. Although this is an important aspect of trust models, such as Mayer et al.'s (1995) model, the trustee's behavior on the trustor's perceptions remain an under-studied aspect of trust research. The current study helped to remedy this issue in the literature.

\section{Checkmate task}

The Checkmate task is a useful scenario for assessing nondichotomous trust behaviors. Indeed, $56 \%$ of the interactions involved a low wager by the Banker, which would indicate a low trusting condition, despite an honest behavior by the GP. Participants did, however, engage in more risky wagering strategies over time. This is in contrast to previous research on dichotomous trust games that typically finds a trust behavior rate of approximately $60-85 \%$ (Wong \& Hong, 2005). One possible reason for the difference in trust behavior may be the larger amounts of money at stake in the current study. Yet, Johnson and Mislin (2011) did not find that endowment size influences trust. It is possible that the added ambiguity of one's true intent masked potential biases of social pressures, making the behavior a truer representation of one's behavior. The Checkmate task may allow for deception on the part of the GP, thus injecting higher uncertainty in the task because the appearance of a distrusting behavior may be driven by factors outside of the control of the trustee, making trustor judgments opaque. Further, Alarconet al (2016) found defection rates of only $5.2 \%$ for familiar dyads and $18.1 \%$ for unfamiliar dyads in a Prisoner's Dilemma study where US $\$ 75$ was at stake in each interaction. Thus, the high rates of trust behavior found in the literature may be slightly overstated once social pressures are reduced.

In addition, the Checkmate task more accurately assesses trust interactions. Participants in the task were more risk averse to losing their initial endowment gained earlier in the study, as illustrated by the low wager condition being the most frequent. This supports Tversky and Kahneman's (1992) prospect theory. However, when faced with trust behaviors from the partner, bankers had a higher likelihood of leaving the low-wager condition, indicating risk adversity had decreased due to the partner's honest behaviors. Thus, unlike previous trust games that focus solely on gain or loss, the Checkmate scenario is more ecologically valid as it entails potential for both, as most trust decisions do.

Another advantage of the task is that it provides a more comprehensive perspective on the trustworthiness construct by incorporating influences not just driven by one's intent (e.g., benevolence and integrity) as in traditional trust games but also inclusive of one's ability. Checkmate affords ambiguity of both partners' behavior and intent. The Banker's behavior is ambiguous as the wager conditions overlap. The GP may receive US\$5 to run the task, but may not know if the Banker placed a Low or Medium wager. In contrast, the GP may want to reciprocate a trusting behavior from the Banker but may not have the opportunity due to low ability. As such, the Checkmate task allows researchers to more accurately capture trust actions, intentions, and beliefs in one comprehensive task. The behaviors are interpreted by each partner, in contrast to classic games such as Prisoner's Dilemma, which allows for absolute knowledge of the partner's behavior. The presence of this ambiguity should create a "weak" situation and thus invoke more direct effects for trust (Dirks \& Ferrin, 2001).

\section{Multivariate multilevel survival analysis}

Complex psychological processes that rely on the interaction of two or more parties are often hard to model. As one participant changes from an emotional state, cognitive state, or some combination of the two, modeling the changes in the interaction of the dyad become significantly harder with traditional approaches. Research has often focused on one aspect of the dyad and modeled those effects (trustworthiness, Jones \& Shah, 2016; trust, Colquitt \& Rodell, 2011), often neglecting behavior. MMSA allows researchers to fully model the complex interaction that occurs in dyads. Although the temporal intervals in the current study were controlled by the experimenter (one trust decision per round of checkmate), MMSA has been used to model real-time changes in affect in parent-child interactions (Stoolmiller \& Snyder, 2014). The methodology offers researchers a new tool for analyzing research and closer approximating dyadic models in the psychological literature.

\section{Limitations and future research}

The current study is not without limitations. First, the task was not experimental; although the current study captures the complexities of real interactions, it does make it difficult to model all interactions as indicated by the low occurrences of I and D behaviors. Indeed, the I models were not able to converge because of the low occurrences in each category $(\mathrm{H}, \mathrm{M}, \mathrm{L})$. Future research may want to manipulate trust games to 
explore I and D more closely. Second, a single-item measure was used to assess trustworthiness. Although previous research indicates single-item measures may be appropriate (Ferrin et al., 2006; Jones \& Shah, 2016), it fails to capture the subtle complexities of trustworthiness. Partners may have assessed their partners in terms of ability, benevolence, and integrity differently than global trustworthiness. This is especially relevant in the novel task type in the current study. Future research should replicate the current study with established measures of ability, benevolence, and integrity to determine the independent effect of each.

Future research could be used to tease apart the unique effects of each trustworthiness facet on trust intentions and behaviors. First, it would be interesting to replicate this study using measures for all three trustworthiness dimensions to examine which facet benefits the most in the face of trusting behaviors and which suffer following either dishonest or inability behaviors. Second, it would be interesting to examine the unique effects of these dimensions in predicting behavior over time. It is possible for instance, that integrity and benevolence would be most predictive of trust behaviors early on in the exchange as partners feel each other out to understand their intentions. Yet, once an understanding of one's intentions is established, the perception of ability may drive trust behaviors. The authors note that a number of different framing techniques could be used to examine the unique effects of a particular trustworthiness dimension to include the difficulty of the task (ability-focused), the goal-directed behavior of the GP and the implications of such actions whether to be team-oriented or self-focused given differential weights for particular performance elements (benevolence -focused), or consistency with values (integrity-focused). Finally, research could examine the impact of the trustworthiness dimensions for those high and low on propensity to trust. It is possible that those high in propensity to trust may not care as much about integrity or benevolence but they may consider ability as the key trust antecedent in these scenarios. Such information would be useful for the trust literature and would help to feed guidelines for effective teams/dyads in organizations.

Author Notes This research is based upon work supported by the Office of the Director of National Intelligence (ODNI), Intelligence Advanced Research Projects Activity (IARPA), via the Air Force Research Laboratory. The views and conclusions contained herein are those of the authors and should not be interpreted as necessarily representing the official policies or endorsements, either expressed or implied, of the ODNI, IARPA, or the US Government. The US Government is authorized to reproduce and distribute reprints for Governmental purposes notwithstanding any copyright annotation thereon.

\section{Appendix}

$$
\begin{aligned}
& \log \left[h_{i}(t)\right]=\left\{\log \left[h_{H H}(t)\right]+\beta_{0,1} P T_{i}+b_{0 i}\right\} H H_{i}(t)+ \\
& \left\{\log \left[h_{L H}(t)\right]+\beta_{1,1} P T_{i}+b_{1 i}\right\} L H_{i}(t)+\left\{\log \left[h_{H D}(t)\right]+\beta_{2,1}\right. \\
& \left.P T_{i}+b_{2 i}\right\} H D_{i}(t)+\left\{\log \left[h_{M D}(t)\right]+\beta_{3,1} P T_{i}+b_{3 i}\right\} M D_{i}(t)+ \\
& \left\{\log \left[h_{L D}(t)\right]+\beta_{4,1} P T_{i}+b_{4 i}\right\} L D_{i}(t)+\left\{\log \left[h_{H I}(t)\right]+\beta_{5,1} P T_{i}\right. \\
& +b_{5 i} H H_{i}(t)+\left\{\log \left[h_{M I}(t)\right]+\beta_{6,1} P T_{i}+b_{6 i}\right\} M I_{i}(t)+ \\
& \left\{\log \left[h_{L I}(t)\right]+\beta_{7,1} P T_{i}+b_{7 i}\right\} L I_{i}(t) .
\end{aligned}
$$

The model equation above depicts the equation for the latent variable of the log hazard rate with $\mathrm{MH}$ as a start state for $\log \left[h_{i}(t)\right]$, which is comprised of the log hazards of all other possible transition states $\log \left[h_{X X}(t)\right] ; i$ indicates the subject or banker; $t$ indicates time within an episode; $\beta$ is fixed regression weight to be estimated [propensity to trust's (PT) effect is being estimated above] the log hazards, and $b$ is the random effects across dyads. The two-letter abbreviation for the dyad state refers to the dyad states displayed in Table 1 (i.e., HH, MH, etc). The dummy variable at the end of each log hazard component is an indicator for each type of transition.

Note: Although the current study only modeled honest behaviors $(\mathrm{HH}, \mathrm{MH}$, and $\mathrm{LH})$, the other states are all possible and thus are part of the final equation for the latent variable and the probability of time within an episode. In addition, more complex models such as mediation are available. For more information on possible equations and uses see Stoolmiller and Snyder (2006, 2014).

\section{References}

Ajzen, I. (1991). The theory of planned behavior. Organizational Behavior and Human Decision Processes, 50, 179-211

Alarcon, G. M., Lyons, J. B., \& Christensen, J. C. (2016). The effect of propensity to trust and familiarity on perceptions of trustworthiness over time. Personality and Individual Differences, 94, 309-315.

Axelrod, R. M. (1984). The Evolution of Cooperation. New York, NY: Basic Books.

Berg, J., Dickhaut, J. W., \& McCabe, K. A. (1995). Trust, reciprocity, and social history. Games and Economic Behavior, 10, 122-142.

Blau, P. M. (1964). Justice in social exchange. Sociological Inquiry, 34, 193-206.

Colquitt, J. A., \& Rodell, J. B. (2011). Justice, trust, and trustworthiness: A longitudinal analysis integrating three theoretical perspectives. Academy of Management Journal, 54, 1183-1206.

Colquitt, J. A., Scott, B. A., \& LePine, J. A. (2007). Trust, trustworthiness, and trust propensity: A meta-analytic test of their unique relationships with risk taking and job performance. Journal of Applied Psychology, 92, 909-927.

Deutsch, M. (1958). Trust and suspicion. Journal of Conflict Resolution, 2, 265-279.

Dietz, G., \& Fortin, M. (2007). Trust and justice in the formation of joint consultative committees. International Journal of Human Resource Management, 18, 1159-1181.

Dirks, K. T. (1999). The effects of interpersonal trust on work group performance. Journal of Applied Psychology, 84, 445-455. 
Dirks, K.T., \& Ferrin, D.L. (2001). The role of trust in organizational settings. Organizational Science, 12, 450-467.

Ferrin, D. L., Bligh, M. C., \& Kohles, J. C. (2007). Can I trust you to trust me? A theory of trust, monitoring, and cooperation in interpersonal and intergroup relationships. Group \& Organization Management, $32,465-499$.

Ferrin, D. L., \& Dirks, K. T. (2003). The use of rewards to increase and decrease trust: Mediating processes and differential effects. Organization Science, 14, 18-31.

Ferrin, D. L., Dirks, K. T., \& Shah, P. P. (2006). Direct and indirect effects of third-party relationships on interpersonal trust. Journal of Applied Psychology, 91, 870-883.

Foddy, M., Platow, M. J., \& Yamagishi, T. (2009). Group-based trust in strangers: The role of stereotypes and expectations. Psychological Science, 20, 419-422.

Gill, H., Boies, K., Finegan, J. E., \& McNally, J. (2005). Antecedents of trust: Establishing a boundary condition for the relation between propensity to trust and intention to trust. Journal of Business and Psychology, 19, 287-302.

Hox, J. J. (2002). Multilevel Analysis: Techniques and Applications. New York, NY: Routledge/Taylor \& Francis Group.

Jarvenpaa, S. L., Knoll, K., \& Leidner, D. E. (1998). Is anybody out there? Antecedents of trust in global virtual teams. Journal of Management Information Systems, 14, 29-64.

Johnson, N. D., \& Mislin, A. A. (2011). Trust games: A meta-analysis. Journal of Economic Psychology, 32, 865-889.

Jones, S. L., \& Shah, P. P. (2016). Diagnosing the locus of trust: A temporal perspective for trustor, trustee, and dyadic influences on perceived trustworthiness. Journal of Applied Psychology, 101, $392-414$.

Kline, R. B. (2005). Principles and practice of structural equation modeling. New York, NY: Guilford Press.

Korsgaard, M. A., Brodt, S. E., \& Whitener, E. M. (2002). Trust in the face of conflict: The role of managerial trustworthy behavior and organizational context. Journal of Applied Psychology, 87, 312319.

Kramer, R. M. (1999). Trust and distrust in organizations: Emerging perspectives, enduring questions. Annual Review of Psychology, 50, 569-598.

Kreps, G. L. (1990). Organizational Communication: Theory and Practice. New York, NY: Longman.

Levin, D., Whitener, E., \& Cross, R. (2006). Perceived trustworthiness of knowledge sources: The moderating impact of relationship length. Journal of Applied Psychology, 91, 1163-1171.

Lewicki, R. J., \& Bunker, B. B. (1995). Trust in relationships: A model of development and decline. In B. B. Bunker, J. Z. Rubin (Eds.), Conflict, Cooperation, and Justice: Essays Inspired by the Work of Morton Deutsch (133-173). San Francisco, CA: Jossey-Bass.

Mayer, R. C., \& Davis, J. H. (1999). The effect of the performance appraisal system on trust for management: A field quasi-experiment. Journal of Applied Psychology, 84, 123-136.

Mayer, R. C., Davis, J. H., \& Schoorman, F. D. (1995). An integrative model of organizational trust. Academy of Management Review, 20, 709-734.

McKnight, D. H., Cummings, L. L., \& Chervany, N. L. (1998). Initial trust formation in new organizational relationships. The Academy of Management Review, 23, 473-490.
Muthén, L. K., \& Muthén, B. O. (1998-2011). Mplus User's Guide. Sixth Edition. Los Angeles, CA: Muthén \& Muthén.

Parkhe, A., \& Miller, S. R. (2000). The structure of optimal trust: A comment and some extensions. The Academy of Management Review, 25, 10-11.

Pillutla, M. M., Malhotra, D., \& Murnighan, J. K. (2003). Attributions of trust and the calculus of reciprocity. Journal of Experimental Social Psychology, 39, 448-455.

Sackett, P. R., \& Larson, J. J. (1990). Research strategies and tactics in industrial and organizational psychology. In M. D. Dunnette, L. M. Hough (Eds.), Handbook of Industrial and Organizational Psychology, Vol. 1 (2nd ed.) (419-489). Palo Alto, CA: Consulting Psychologists Press.

Schaubroeck, J. M., Peng, A. C., \& Hannah, S. T. (2013). Developing trust with peers and leaders: Impacts on organizational identification and performance during entry. Academy of Management Journal, $56,1148-1168$.

Schoorman, F. D., Mayer, R. C., \& Davis, J. H. (1996). Organizational trust: Philosophical perspectives and conceptual definitions. The Academy of Management Review, 21, 337-340.

Serva, M. A., Fuller, M. A., \& Mayer, R. C. (2005). The reciprocal nature of trust: A longitudinal study of interacting teams. Journal of Organizational Behavior, 26, 625-648.

Shapiro, D. L., Sheppard, B. H., \& Cheraskin, L. (1992). Business on a handshake. Negotiation Journal, 8, 365-377.

Simpson, J. A. (2007). Psychological foundations of trust. Current Directions in Psychological Science, 16, 264-268.

Stoolmiller, M., \& Snyder, J. (2006). Modeling heterogeneity in social interaction processes using multilevel survival analysis. Psychological Methods, 11, 164-177.

Stoolmiller, M., \& Snyder, J. (2014). Embedding multilevel survival analysis of dyadic social interaction in structural equation models: Hazard rates as both outcomes and predictors. Journal of Pediatric Psychology, 39, 222-232.

Tabachnick, B. G., \& Fidell, L. S. (2013). Using multivariate statistics. Boston, MA: Pearson/Allyn \& Bacon.

Thaler, R. H., \& Johnson, E. J. (1990). Gambling with the house money and trying to break even: The effects of prior outcomes on risky choice. Management Science, 36, 643-660.

Therneau, T. M., \& Grambsch. (2000). Modeling survival data: Extending the Cox model. New York, NY: Springer.

Tversky, A., \& Kahneman, D. (1992). Advances in prospect theory: Cumulative representation of uncertainty. Journal of Risk and Uncertainty, 5, 297-323.

Wanous, J. P., Reichers, A. E., \& Hudy, M. J. (1997). Overall job satisfaction: How good are single-item measures? Journal of Applied Psychology, 82, 247-252.

Weber, J. M., Malhotra, D., \& Murnighan, J. K. (2004). Normal acts of irrational trust: Motivated attributions and the trust development process. Research in Organizational Behavior, 26, 75-101.

Wedekind, C., \& Milinski, M. (1996). Human cooperation in the simultaneous and the alternating prisoner's dilemma: Pavlov versus generous tit-for-tat. Proceedings of the National Academy of Sciences of the United States of America, 93, 2686-2689.

Wong, R. Y. M., \& Hong, Y. Y. (2005). Dynamic influences of culture on cooperation in the prisoner's dilemma. Psychological Science, 16, 429-434. 